\title{
Development of wall inspection robots
}

\author{
A.NISHI, H.MIYAGI and K.ISHIHARA \\ Faculty of Engineering, Miyazaki University \\ Gakuen-Kibanadai, Miyazaki 889-21 \\ JAPAN
}

\begin{abstract}
Use of wall-climbing robot for inspection or maintenance of wall surfaces has been anticipated for a long time. Four quite different models have been developed in our laboratory. The present model can move on a wall by using the thrust force of propellers and can fly whenever it is required. Its mechanism and control system are discussed.
\end{abstract}

\section{INTRODUCTION}

A robot capable of moving on a vertical wall has been looked forward to a long time. It could be used for wall inspection, wall repair, rescue, fire-fighting and so on. Four quite different types of wall-climbing robot models have been developed in our laboratory over the last 20 years. The first model, shown in Fig.1, has a large sucker and crawlers as a moving mechanism. [1] Recently, many kinds of this type model have been developed for wall inspection in Japan.[2]-[4] The second type, shown in Fig.2, is a biped walking model, which has a small sucker on each foot. As this can be applied to almost all irregular wall surfaces, it has a wider applicability than the former.

In general, the walking motion is not very fast so that the walking model takes much time to climb up to higher places on the wall. A robot would be required to climb up to higher places on buildings in a short time for the quick inspection and/or emergency purposes. The third model, shown in Fig.3, aims at these purposes. It has propellers, and their thrust forces are inclined a little toward the wall to make use of the frictional force between the wheels and the wall surface, as well as to support the robot itself.[6]-[9] Sometimes unexpected strong winds occur on the wall of high-rise buildings. In such cases, the control system to compensate for the wind load is important to avoid having the robot falling from the surface. This situation has been previously discussed in detail in Refs. [6] and [7].

On the other hand, often there are many obstacles at the lower part of buildings, such as trees, eaves, entrances, and so on. In such cases, it would be useful if the robot could fly over these obstacles and can reach the upper wall surface. Moreover, if the 


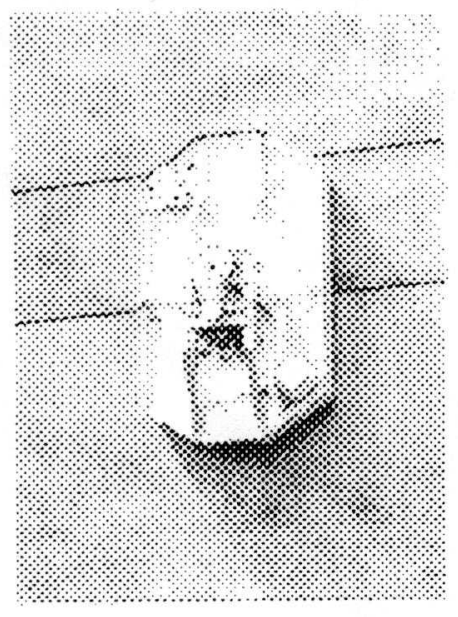

Fig. 1 Large sucker model.

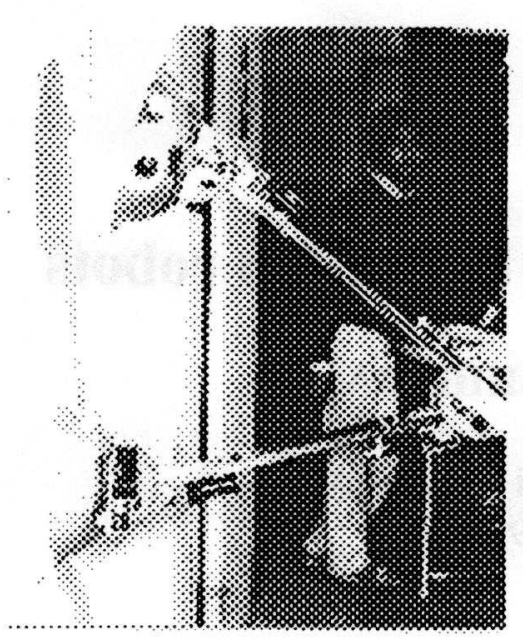

Fig.2 Biped walking model.

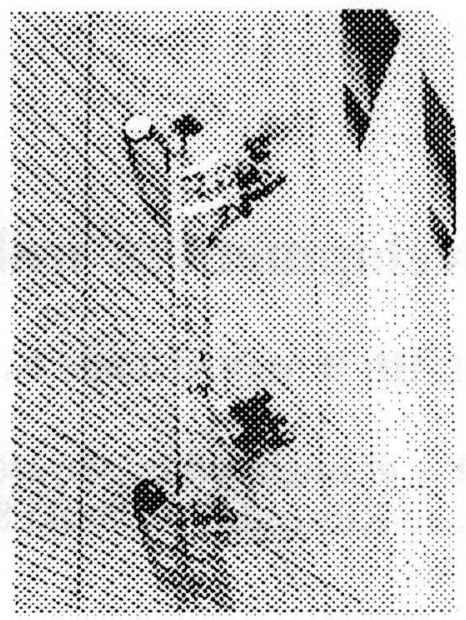

Fig.3 Propeller type model.

robot falls from a higher place on the wall surface by accident, it needs to make a soft landing to avoid endangering itself or its surroundings. These maneuvers can be done by employing a mechanism and control system enabling it to fly. As the robot has an enough thrust force to sustain itself in the air, it can fly or land. This is the fourth type model and its mechanism and control system are discussed in this paper mainly.[10][12]

\section{MECHANISM OF THE FLIGHT ROBOT}

An example of a conceptual model is shown in Fig.4. A combination of an engine and propeller is used as a main thrustor of the robot. To use a robot for carrying a TV camera for the inspection or other tools to repair the wall surface, it is necessary to reach and attach itself to the wall surface at the lower part of buildings. However, often there are obstacles such as trees, eaves and entrances at the lower part of the buildings. In such cases, it may be difficult for the robot to attach itself to the wall directly. As the robot concerned here, has enough thrust force to lift itself up in the air, it can fly over these obstacles employing a suitable mechanism and control system.

Almost the same situation will occur at higher parts of the buildings, where there are eaves or steps on the wall surface which are difficult to get over using the conventional moving mechanism.

Moreover, there is danger of the robot falling from the wall surface by an unexpected strong wind or accident. In such cases, the robot must make a soft landing, or the fall would be very dangerous for the robot itself and its surroundings. These situations can be avoided by designing the robot so that it is able to fly or land. These maneuvering is shown in Fig. 5 schematically.

However, its major purpose is not to fly like a helicopter, but to move on a wall surface using its wheels, since moving on a wall is safer. Therefore, it has a long body with many wheels, and its thrust force has a component toward the wall to produce 

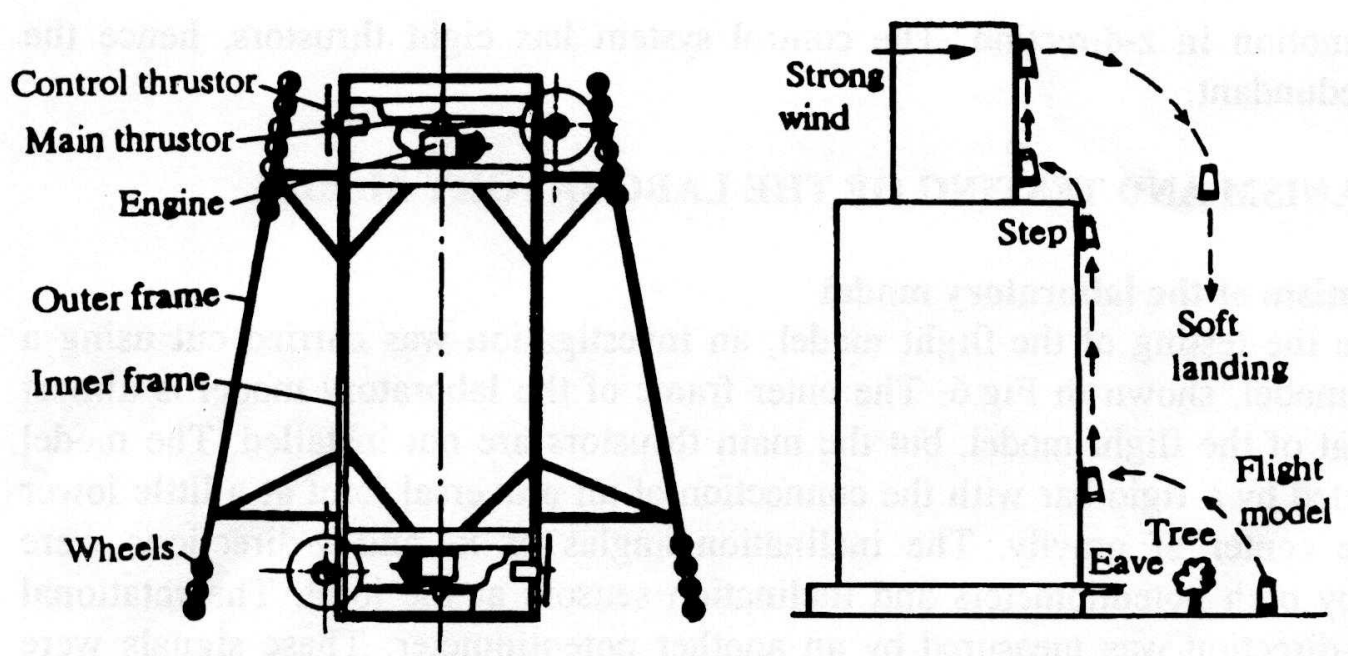

Fig.4 Conceptual model. Fig.5 Maneuvering of the flight model.

frictional force between the wheels and the wall surface. The conceptual robot is designed for this requirement. Two main propellers are installed at the top and bottom of the body to lift the robot up in the air. Eight control propellers are employed to control the robot in the air and to produce the force toward the wall when it is moving on the wall surface.

\section{CONTROL SYSTEM OF THE FLIGHT MODEL}

There are many methods to produce the control force in the air. The up and down motion of the robot is done by controlling the thrust force, i.e., the control of revolutional speed of each engine.

A few methods were examined to accomplish the flight control of the flight model. As the flight model is symmetrical along the main thrust axis, a righting force is not produced. So, the mechanism to produce it is important. First was a method of inclining the thrust force directly. This method could be produced large side force, however, its mechanism was heavier, so that it was inferior for the flight model and was difficult to control the robot quickly. Second was the method to incline the propeller slip stream by the vanes. This mechanism was lighter than the former, but the non-linear relations appear between the forces, so that the design of the control system was difficult. Third is the method to employ the many control thrustors, which is used on the present model. Eight propellers are used as the control thrustors. Each thrustor is controlled independently. A set of four control thrustos is arranged upper and lower parts of the model, shown in Fig.4. The tail rotors of the hobby helicopter model are employed. Each rotor is driven by a small DC motor and the pitch angle of the blade is controlled by an individual servo-motor. The forward or backward thrust force is produced.

The flight model has 5-DOF in the air except for up and down motion. They are side movement and inclinations in $\mathrm{x}$ - and $\mathrm{y}$-directions on the horizontal plane, and the 
rotational motion in $\mathrm{z}$-direction. The control system has eight thrustors, hence the system is redundant.

\section{MECHANISM AND TESTING OF THE LABORATORY MODEL}

\subsection{Mechanism of the laboratory model}

Before the testing of the flight model, an investigation was carried out using a laboratory model, shown in Fig.6. The outer frame of the laboratory model is almost same as that of the flight model, but the main thrustors are not installed. The model was supported by a rigid bar with the connection of an universal joint at a little lower part of the center of gravity. The inclination angles of $x$ - and $y$-directions were measured by both potentiometers and inclination sensors at the joint. The rotational angle in $\mathrm{z}$-direction was measured by an another potentiometer. These signals were sent by wire to the computer. The feedback signals from the computer to the model were given by the radio signals. These were the signals of the pitch control of thrustor blades. Eight channels radio control system was used for the feedback control.

\subsection{Control method and test results}

At first, each control thrustor was driven at a constant speed with neutral pitch angle. When the pitch angle is controlled, it can produce the thrust force forward or backward direction corresponding to the blade angle. The state feedback control method is applied to the force control of each thrustor. As the inclination sensor has a certain amount of time lag, a phase lead compensation is examined.

A few examples of test results are shown in Fig.7 and 8. In Fig.7 the step responses of inclination angles of $\mathrm{x}$-direction are presented. For a suitable pole

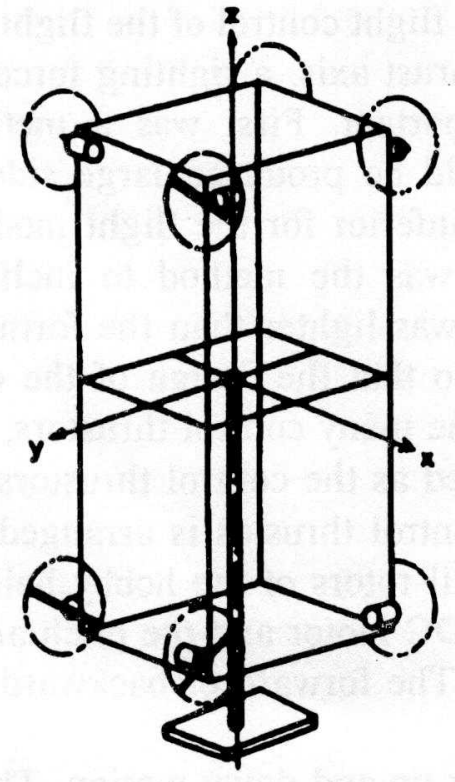

Fig.6 Laboratory model.

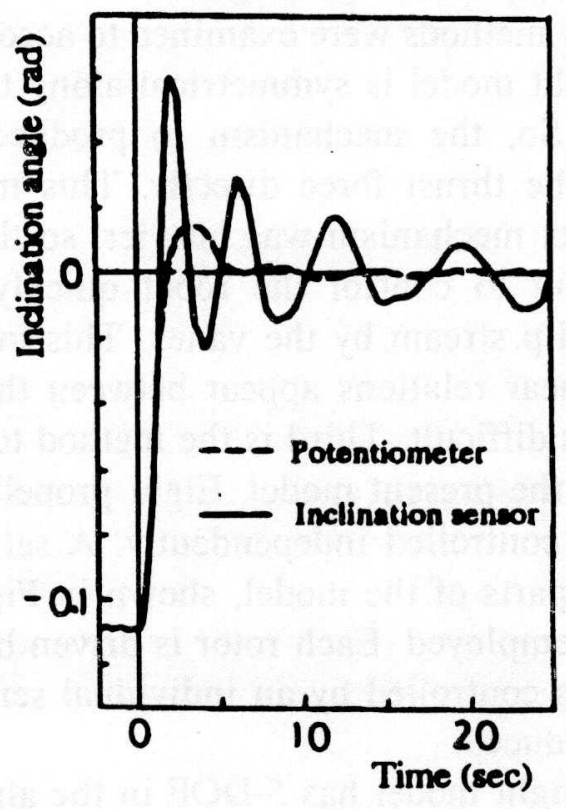

Fig.7 Step responses. 
distribution of the transfer function, the responses for the potentiometer and inclination sensor signals are compared, and it is shown clearly that more sensitive response can be obtained by using the potentiometer signal. The laboratory model can be controlled manually, that is, the inclination of the model can be controlled by adding a suitable voltage on the feedback control signals by the stick operation of the radio controller. The results of stick operation and the response is shown in Fig.8.

\section{FLIGHT SIMULATION}

Before the construction of the flight model, the flight simulation was carried out to ascertain the moving of the flight model in the air, by using the laboratory model. The side movement of the flight model occurs by the side force component produced by the inclination of the main thrust force. This motion was created in the computer and combined with the motion of the laboratory model. The whole motion of the free flight in the air of the flight model could be seen on the CRT of the computer. The moment of inertia of the laboratory model was adjusted almost same as that of the flight model in any direction, so the maneuvering of the flight model was realistic. The motion was controlled by the stick operation of the radio controller.

\section{TESTING OF THE FLIGHT MODEL}

The last stage of the development is the construction of the flight model and its testing. There were, however, many difficult problems on the way of development. They were the force balance between the main thrust force and the whole weight of the model, the performance of the power source of the control thrustors, the noise

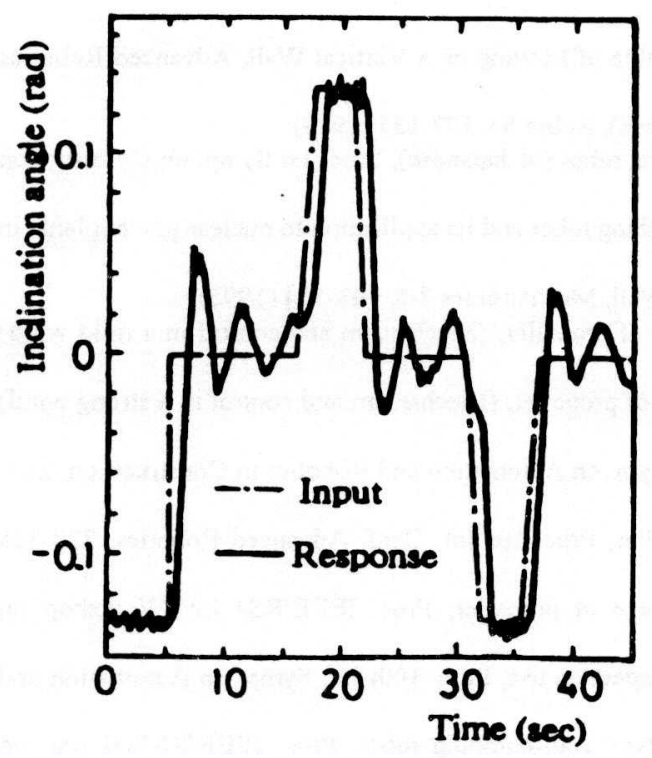

(a) Inclination sensor.

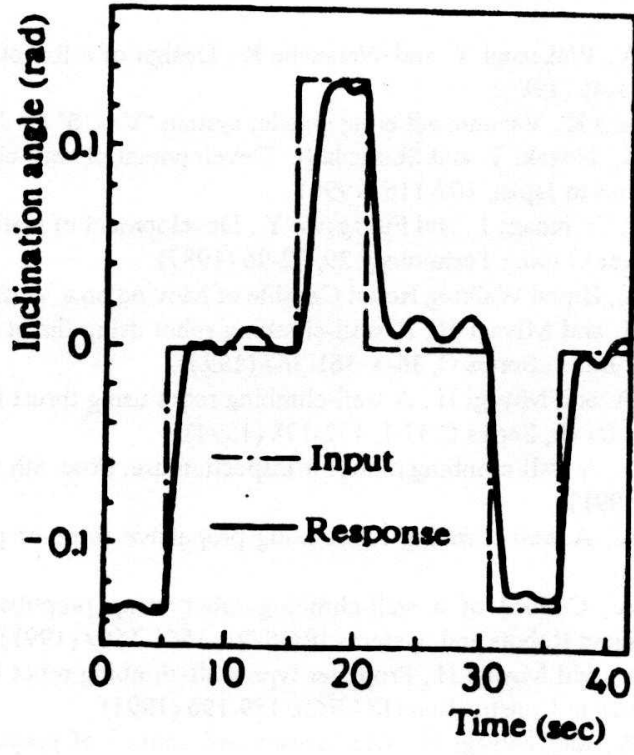

(b) Potentiometer.

Fig.8 Responses of the laboratory model. 
production of the ignition plug and high voltage parts, and so on. These were solved step by step, and the flight model is now under constructing.

\section{CONCLUSION}

Four different types of wall-climbing robots have been developed in out laboratory. The first model had a large sucker and could be used on flat and wide wall surfaces. The second was a biped walking model, which had a small sucker on each foot. This could be applied to almost all irregular wall surfaces. The third model could move on a vertical wall at high speed. It had propellers and the thrust force is inclined a little toward the wall to produce the frictional force between the wheels and wall surface, as well as the lifting force to sustain the robot itself.

When the third model is employed for inspection and/or repair of the wall surface, at first it is necessary for it to reach and attach itself to the wall surface. Often there are obstacles at the lower part of the buildings, such as eaves, trees, entrances, etc.. Therefore, it would be very useful if the robot could fly over these obstacles and reach the upper wall surface. Moreover, if the robot falls from the wall surface by accident, a soft-landing control has to be employed to avoid danger to the robot and surroundings. For these purposes a flight model has been considered. The mechanism and control systems for such maneuvering have been discussed. As a result, it was demonstrated that the laboratory model could be controlled easily, and similar control methods can be applied to the flight model.

The flight model is under constructing. The next step is the flight test in the air. This is now in preparation.

\section{REFERENCE}

[1] Nishi A., Wakasugi Y. and Watanabe K., Design of a Robot Capable of Moving on a Vertical Wall, Advanced Robotics $1-1,33-45(1986)$

[2] Nagatsuka K., Vacuum adhering crawler system "VACS" (in Japanese), Robot 53, 127-135 (1989).

[3] Ikeda K., Nozaki T. and Shimada S., Development of wall-climbing robot (in Japanese), Proc. 1st Symp. on Construction Robotics in Japan, 107-116(1990)

[4] Sato K., Tominaga I., and Fukagawa Y., Development of wall-climbing robot and its application to nuclear power plant (in Japanese).Piping Technology 29, $92-96$ (1987).

[5] Nishi A., Biped Walking Robot Capable of Moving on a Vertical Wall, Mechatronics 2-6, 543-554 (1992).

[6] Nishi A. and Mlyagi H., A wall-climbing robot using thrust force of propeller, (Mechanism and control in a mild wind), JSME Int. J., Series C, 36-3, 361-367 (1993).

[7] Nisihi A. and Miyagi H., A wall-climbing robot using thrust force of propeller, (Mechanism and control in a strong wind), JSME Int. J., Series C 37-1, 172-178 (1994).

[8] Nishi A., A wall-climbing robot for inspection use, Proc. 8th Int. Sym. on Automation and Robotics in Constructi on, 267. 274 (1991).

[9] Nishi A., A wall-climbing robot using propulsive force of propeller, Proc. Sth Int. Conf. Advanced Robotics, 320-325 (1991).

[10] Nishi A., Control of a wall-climbing robot using propulsive force of propeller, Proc. IEEE/RSJ Int. Workshop on Intelligent Robots and Systems IROS '91, 1561-1567 (1991).

(11) Nishi A., and Miyagi H., Propeller type wall-climbing robot for inspection use, Proc. 10th Int. Symp. on Automation and Robotics in Construction (ISARC), 189-196 (1993).

[12] Nishi A., and Miyagi H., Mechanism and control of propeller type wall-climbing robot, Proc. IEEE/RSJ/GI onf. on Intelligent Robots and Systems, IROS'94, 1724-1729 (1994). 